\title{
Mejoramiento Genético y Adaptación de Genotipos y Clones Superiores de Jojoba Sinmondsia chinensis Link (Scheneider) en Zonas Áridas Salinas de Tacna
}

\section{Genetic Improvement and Adaptation of Genotype and Superior Clones of Jojoba Sinmondsia chinensis Link (Scheneider) in Arid Zones Salinas from Tacna}

${ }^{1}$ Nelly Arévalo Solsol, 2 Óscar Fernández Cutire

\section{RESUMEN:}

Se establecieron 280 plantas de Jojoba proveniente de un germoplasma de alta variabilidad genética. Los objetivos fueron: Evaluar y seleccionar genotipos de jojoba en condiciones árido salinas de Tacna. La metodología para la obtención de estos genotipos fueron en t etapas: lera Etapa: Identificación de plantas madres de buena arquitectura, alta producción de semillas, tolerantes a plagas y enfermedades, evaluados por dos años. En la 2da Etapa: Obtención de plantones de 25 a $30 \mathrm{~cm}$, bajo condiciones de invernadero. En la 3era Etapa: Se trasplantó a campo definitivo 280 plantones (provenientes de la segunda etapa), las cuales fueron seleccionadas por vigor de plantas y estado sanitario. En la tra Etapa: evaluación y selección de genotipos durante un periodo de 8 años y evaluación de clones durante 4 años. Los genotipos fueron seleccionados por su producción de semillas, arquitectura de plantay por sanidad, recomendándose a genotipos: JLP45 que alcanzó $1414.73 \mathrm{~g} ;$ JLP-57 con $1242.02 \mathrm{~g} ;$ JLP-25 con $1216.15 \mathrm{~g}$. Los clones que destacaron fueron: $\mathrm{C} 1-11 ; \mathrm{C} 5-46, \mathrm{C} 2-21$.

Palabras Clave: genotipos, selección, adaptación, clon, variabilidad genética.

\section{ABSTRACT:}

280 jojoha plants, coming from a high genetic variability germplasm were established. Objectives were to: assess and choose genotypes of jojoha in arid saline conditions in Tacna. The methodology for obtaining these genotypes were in 4 stages: Ist stage: identification of mother plant with good architecture, high production of seeds, tolerant to pests and diseases, evaluated for two years. $2 \mathrm{Nd}$ stage: attainment of plants from 25 to $30 \mathrm{~cm}$ under greenhouse conditions. In the 3rd stage: 280 (from phase II) plants, were transplantes to final field. then were selected by force of plants and sanitary state. In the th stage: evaluation and selection of genotypes during a period of 8 years and evaluation of clones for 4 years. Genotypes were selected for its seed production, architecture of plant and health, recommending 3 genotypes: JLP-45 reached $1414.73 \mathrm{~g} ;$ JLP-57 with $1242.02 \mathrm{~g}$; JLP-25 with $1216.15 \mathrm{~g}$. Clones that highlighted were: $\mathrm{C} 1-11$; C5-46, C2-21.

Keywords: genotypes, selection, adaptation, clon, genetic variability.

\footnotetext{
' Magister Scientae en Mejoramiento Genético de Plantas, Magister en Docencia Universitaria y Gestión Educativa. Ingeniero Agrónomo. Facultad de Ciencias Agropecuarias. Universidad Nacional Jorge Basadre Grohmann

${ }^{2}$ Doctor en Filosofia, Ph. D. en Ciencias Agricolas, Master of Science in Agriculture, Ingeniero Agrónomo.

Facultad de Ciencias Agropecuarias. Universidad Nacional Jorge Basadre Grohmann
} 


\section{INTRODUCCIÓN}

La Jojoba Sinmondsia chinensis Link (Scheneider), es un arbusto perenne, árido-activo, pertenece a la familia de las Buxáceas caracterizándose por ser el único género xerofitico de la especie, son plantas dioicas y longevas. La polinización es anemófila, los frutos (cápsulas) se obtienen a los 6 meses después de la fecundación en las plantas femeninas, observándose de la 3 semillas/cápsulas, cuyo peso fluctúa de $0.4 \mathrm{~g}$ hasta $1.5 \mathrm{~g}$. Las semillas contienen cera liquida de $40 \%$ a $60 \%$. Tiene una amplia gama de usos, desde la industria de cosméticos hasta la fabricación de lubricantes.

Actualmente la producción de Jojoba es utilizada en la industria de cosméticos.

En la actualidad no existe trabajos de investigación tanto a nivel nacional como local en selección de genotipos que tengan un buen comportamiento, en la producción de semillas y tolerancia a estreses bióticos y abióticos, estudios de mercado, entre otros la cual trae como consecuencia poco interés de los agricultores para este cultivo. Frente a esta situación la FCAG de la UNJBG de Tacna planteó esta investigación de Mejoramiento Genético y Adaptación de genotipos y de Clones Superiores de Jojoba Sinmondsia chinensis (Link) Scheneider:

\section{Objetivos}

Evaluar genotipos de jojoba en condiciones árido salinas de Tacna

Seleccionar genotipos superiores de atributos agronómicos deseables para posteriormente clonarlos.

Evaluar clones de jojoba en las condiciones árido salinas de Tacna.

\section{MATERIALES Y MÉTODOS}

\subsection{Ubicación}

El trabajo se instaló en el Centro Experimental Los Pichones (CEA III) de propiedad de la Facultad de Ciencias Agricolas, de la Universidad Nacional Jorge Basadre Grohmann de Tacna, ubicado en la Ciudad Universitaria, distrito, provincia y Región Tacna, cuyas coordenadas geográficas son: $17^{\circ} 59^{\circ} 38^{\prime \prime}$ de latitud sur y $70^{\circ} 1422^{\prime \prime}$ longitud oeste.

El campo experimental donde se instalo fue un terreno árido salino, suelo de origen aluvial con predominio de textura arenosa típico de las zonas y regiones desérticas áridas y semiáridas: con C.E. $\left(\mathrm{ds} / \mathrm{ma} 25^{\circ} \mathrm{C}\right)$ de 5.20 que es considerado suelo moderadamente salino; el pH: 6.8 representa un suelo ligeramente acido.

La materia orgánica de $1.30 \%$ por lo que se considera baja, con respecto al contenido de potasio es considerada alta $370 \mathrm{ppm}$; asimismo el contenido de fosforo de $76.33 \mathrm{ppm}$, CIC de 10.20 que son considerados como medio.

\subsection{Metodología}

En 1995, se establecieron 280 plantas de Jojoba cuyo material genético se obtuvo de un germoplasma de aproximadamente 3000 plantas, proveniente de semilla botánica seleccionada de una plantación comercial de alta variabilidad genética, de la zona de Los Palos, Tacna.

La metodologia para la obtención de estos genotipos fue:

\section{leraEtapa}

Identificación de plantas madres que tenian buena arquitectura, alta producción de semillas, tolerantes a plagas y enfermedades. La recolección de las semillas se efectuó después de una evaluación (por atributos agronómicos) por un periodo de 2 años.

\section{2daEtapa}

Obtención de plantones bajo condiciones de invernadero, logrados de semilla sexual provenientes de las plantas madres identificadas en la primera etapa. la altura promedio fue de 25 a $30 \mathrm{~cm}$ de altura a los 6 meses.

\section{3eraEtapa}

Se trasplantó a campo definitivo 280 plantones (provenientes del material de la segunda etapa), las cuales fueron seleccionadas teniendo en cuenta el vigor de las plantas, estado sanitario de las plantas las cuales fueron plantadas a un distanciamiento de $2 m$ entre surcos y $1 \mathrm{~m}$ entre las plantas.

Aproximadamente a $\operatorname{los} 2$ años cuando las plantas masculinas iniciaban la floración se seleccionaron las de mayor vigor, dejando en la población una relación de 1 polinizante y de 6 plantas femeninas $(1: 6)$

\section{tra Etapa}

Selección y evaluación de genotipos durante un periodo de 6 años.

Los genotipos fueron seleccionados por su producción de semillas, arquitectura de planta y por su sanidad.

Se seleccionaron el $15 \%$ de las plantas femeninas y recomendándose los 3 genotipos de mejores atributos agronómicos.

Las evaluaciones efectuadas en esta etapa fueron las siguientes:

\section{a) Evaluación Morfológica de genotipos de jojoba}

Altura de planta (h) es la distancia tomada desde el nivel del suelo hasta la última rama superior expresada en $\mathrm{cm}$.

Posición de cápsula. Se ha considerado la siguiente escala:

$\mathrm{A}=$ posición alterna; $\mathrm{C}=$ posición continua; $\mathrm{R}=$ racimo Diánietro de follaje. Se midió el diámetro promedio del follaje expresado en $\mathrm{m} 2$. 
Forma de fruto: fue medida según la escala siguiente:

$\mathrm{A}=$ Alargado: $\mathrm{O}=$ Ovalado: $\mathrm{E}=$ Eliptica: $\mathrm{P}=$ Pequeño; $\mathrm{M}=$ Mediano; $\mathrm{G}=$ Pequeño

Forma de hoja medido según la escala siguiente:

$\mathrm{A}=$ Alargado: $\mathrm{O}=$ Ovalado: $\mathrm{E}=$ Eliptico; $\mathrm{G}=$ Grande; $\mathrm{M}=$ Mediano: $\mathrm{P}=$ Grande

Arquitectura de Planta medido según la siguiente escala:

$\mathrm{R}=$ Regular $/$ Planta abierta; $\mathrm{B}=$ Bueno $/$ Planta ligeramente compacta: $\mathrm{MB}=$ Muy Bueno/Planta compacta

\section{b) Producción de Semillas por genotipos}

Se pesó las semillas producidas por cada genotipo. individualmente por año. Solo. se están presentando la produccion de las semillas de los genotipos correspondientes a las campañas 2008 y 2009.

c) Evaluación de clones

Se han instalado clones de jojoba a un distanciamiento de $2 \mathrm{~m}$ entre lineas y $1 \mathrm{~m}$ entre plantas, plantadas a una relación de ! polinizante y 6 hembras."

Se pesó las semillas producidas de los clones. Se presenta la producción de semillas de los clones correspondientes a las campañas 2008 y 2009.

\subsection{Conducción del Experimento}

Durante la investigación anualmente se han realizado las labores agronómicas que a continuación se detallan.

- Deshierbos: Se realizaron 4 deshierbos anualmente, la cual fue realizada manualmente.

- Riegos: se realizó cada 15 dias en verano y cada mes en invierno con un volumen aproximado de 3000 a 4500 $m 3 /$ ha, dichos riegos fueron ligeros.

- Fertilización: La fómula de abonamiento empleada para todas las campañas fue de 80:60:60, N-P-K respectivamente para ello se utilizó el fertilizante compuesto 20-20-20 de N-P-K y se complementó con urea $(46 \% \mathrm{~N})$, se puede indicar que estas plantas responden muy bien al uso de fertilizantes en donde se aprecia un mayor incremento de ramas fruteras especialmente de flores femeninas y frutos. Asimismo se aplicó aproximadamente $1 \mathrm{~kg}$ de estiércol a cada planta

- Control fitosanitario: No se han detectado problema de plagas, se vio ataque de pulgones $A$ phis gossipi en forma localizada. la cual fue controlada mediante lavados de follaje.

- Podas: Se realizó las podas a las plantas femeninas. con la finalidad de inducir mayor número de ramas fruteras (ramas jovenes). Solo se podó plantas que no estaban en floracion, y asi mejorar la estructura de las plantas femeninas. Esta labor se realizó después de la cosecha.

Tabla ${ }^{\circ} 1$. Evaluación Morfologica de Genotipos de .lojoba - Los Pichones 2009

\begin{tabular}{|c|c|c|c|c|c|c|c|c|c|}
\hline \multirow[b]{2}{*}{ Cod. } & \multirow{2}{*}{$\begin{array}{l}\text { Alt. } \\
(\mathbf{c m} .)\end{array}$} & \multirow{2}{*}{$\begin{array}{l}\text { Diámetro } \\
\text { de follaje } \\
\left(\mathrm{m}^{2}\right)\end{array}$} & \multirow{2}{*}{$\begin{array}{c}\text { Arquit. } \\
\text { De } \\
\text { plta. }\end{array}$} & \multirow{2}{*}{$\begin{array}{c}\text { Vigor } \\
\text { de } \\
\text { plta. }\end{array}$} & \multicolumn{2}{|l|}{ Hojas } & \multirow{2}{*}{$\begin{array}{l}\text { Flores } \\
\text { Posic. }\end{array}$} & \multicolumn{2}{|c|}{ Frutos } \\
\hline & & & & & Forma & Tam. & & Tam. & Forma \\
\hline JLP-083 & 230 & 2.7 & MB & $\mathrm{MB}$ & Elíptica & M & $\mathrm{C}$ & G & Ovoide \\
\hline JLP-001 & 186 & 3.42 & B & B & Eliptica & G & C & P & Ovoide \\
\hline JLP-060 & 183 & 2.38 & B & B & Elíptica & $P$ & $\mathrm{C}$ & $P$ & Ovoide \\
\hline JLP-045 & 175 & 1.87 & B & MB & Lanceolada & M & C & P & Ovoide \\
\hline JLP-094 & 170 & 2.08 & B & $\mathrm{R}$ & Oblonga & G & A & P & Ovoide \\
\hline JLP-043 & 170 & 2.08 & $\mathrm{MB}$ & $\mathrm{MB}$ & Oblonga & G & A & $P$ & Ovoide \\
\hline JLP-053 & 168 & 1.96 & B & MB & Elíptica & $P$ & $\mathrm{C}$ & M & Alargada \\
\hline ЛLP-002 & 167 & 2.5 & MB & B & Eliptica & G & A & $\mathrm{P}$ & Ovoide \\
\hline JLP-025 & 166 & 1.4 & B & MB & Eliptica & $P$ & $\mathrm{C}$ & $\mathrm{P}$ & Ovoide \\
\hline JLP-070 & 160 & 1.1 & B & $\mathrm{R}$ & Oblonga & M & C & P & Ovoide \\
\hline JLP-024 & 160 & 1.54 & B & B & Oblonga & M & A & M & Alargada \\
\hline JLP-007 & 155 & 1.4 & B & $\mathrm{R}$ & Eliptica & M & A & $P$ & Ovoide \\
\hline JLP-0 10 & 154 & 1.73 & MB & MB & Elíptica & $P$ & $\mathrm{C}$ & $\mathrm{P}$ & Alargada \\
\hline JLP-236 & 150 & 1.26 & $\mathrm{R}$ & B & Eliptica & M & A & $\mathrm{P}$ & Alargada \\
\hline JLP-057 & 150 & 2.06 & MB & MB & Elíptica & $\mathrm{M}$ & A & M & Ovoide \\
\hline JLP-016 & 150 & 1.43 & B & B & Oblonga & G & $\mathrm{C}$ & M & Alargada \\
\hline JLP-210 & 150 & 3.51 & B & B & Oblonga & G & A & $\mathrm{P}$ & Esférica \\
\hline JLP-202 & 150 & 1.6 & $\mathrm{R}$ & B & Elipticas & M & $\mathrm{C}$ & P & Ovalada \\
\hline JLP-235 & 149 & 2.24 & B & B & Elíptica & M & $\mathrm{C}$ & P & Alargada \\
\hline JLP-236 & 148 & 1.93 & B & MB & Eliptica & M & A & $\mathrm{P}$ & Ovoide \\
\hline JLP-005 & 145 & 1.32 & B & B & Eliptica & M & A & M & Ovoide \\
\hline JLP-012 & 124 & 1.92 & B & B & Oblonga & G & $\mathrm{C}$ & M & Alargada \\
\hline JLP-004 & 120 & 1.68 & B & B & Oblonga & G & $\mathrm{C}$ & $P$ & Alargada \\
\hline JLP-064 & 110 & 0.56 & B & B & Eliptica & $P$ & $\mathrm{C}$ & $P$ & Ovoide \\
\hline JLP-115 & 90 & 0.72 & $\mathrm{R}$ & $\mathrm{R}$ & Elíptica & P & A & M & Alargada \\
\hline JLP-208 & 90 & 0.88 & $\mathrm{R}$ & $\mathrm{R}$ & Oblonga & G & A & G & Alargada \\
\hline PROMEDIO & 152.7 & 1.82 & & & & & & & \\
\hline
\end{tabular}




\section{RESULTADOS}

\subsection{Evaluación morfológica de genotipos}

En la Tabla $\mathrm{N}^{\circ} 01$, se presenta la Evaluación morfológica de los genotipos: altura de plantas $(\mathrm{cm})$, diámetro de follaje $\left(\mathrm{m}^{2}\right)$, arquitectura, vigor de plantas, hojas (forma y tamaño), posición de flores, frutos (tamaño y forma).

La altura promedio es de $152.7 \mathrm{~cm}$ destacando los genotipos JLP 083, JLP 001 y JLP 060, con $230 \mathrm{~cm}, 186$ y $183 \mathrm{~cm}$ respectivamente. En cuanto al diámetro de follaje destaca el genotipo JLP 210 con $3.51 \mathrm{~m}^{2}$, seguido de los genotipos JLP 001 y JLP 235 con $2.38 \mathrm{~m}^{2}$ y 2.24 $\mathrm{m}^{2}$ respectivamente. Los genotipos en su mayoría tienen posición de flores y frutos de tipo continuo, son de buena arquitectura y tienen un buen vigor de planta. Las formas de las cápsulas y la forma de las hojas presentan alta variabilidad en los diferentes genotipos, independientemente de la producción de semillas.

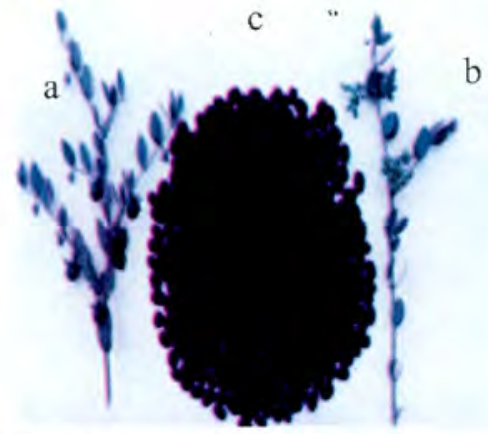

Figura No1. a) Rama femenina b) Rama masculina c) Semillas

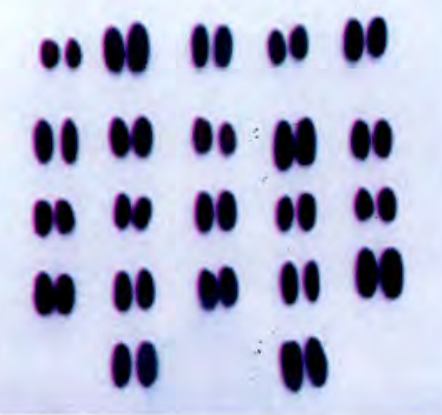

Figura $N^{\circ}$ 2. Formas y tamaños de semillas

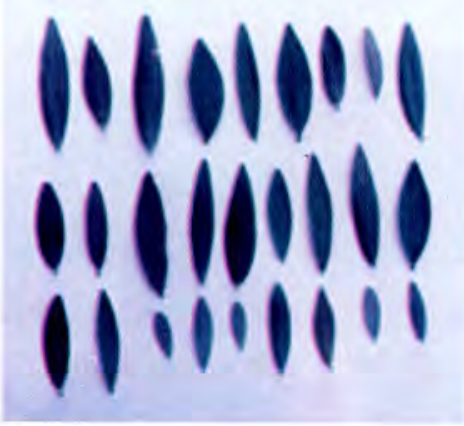

Figura $N^{\circ} 3$. Variabilidad en forma y tamaño de hojas

\subsection{Producción de semillas}

En la Tabla $\mathrm{N}^{\circ} 2$ y Tabla $\mathrm{N}^{\circ} 3$ se reporta la producción de semillas y número de semillas por genotipo en el año 2008 y 2009 respectivamente.

Tabla $\mathbf{N}^{0}$ 2: Producción de semillas y número de semillas por genotipo. Tacna 2008

\begin{tabular}{|c|c|c|c|c|}
\hline Order & Genotipo & $\begin{array}{l}\mathrm{N}^{\circ} \text { de } \\
\text { sem. }\end{array}$ & $\begin{array}{l}\text { Peso Sem. } \\
\text { (g) }\end{array}$ & $\begin{array}{c}\text { Peso } 100 \\
\text { Sem. }\end{array}$ \\
\hline 1 & JLP - 202 & 1708 & 1737.50 & 106.00 \\
\hline 2 & JLP - 25 & 1895 & 1720.40 & 101.70 \\
\hline 3 & ЈLP - 57 & 1560 & 1295.30 & 95.80 \\
\hline 4 & JLP - 53 & 1156 & 1104.50 & 111.40 \\
\hline 5 & JLP - 45 & 838 & 884.50 & 110.90 \\
\hline 6 & JLP - 16 & 749 & 878.30 & 134.90 \\
\hline 7 & JLP - 10 & 880 & 872.90 & 105.90 \\
\hline 8 & JLP - 210 & 860 & 840.00 & 105.60 \\
\hline 9 & JLP - 98 & 636 & 763.30 & 121.80 \\
\hline 10 & JLP - 115 & 939 & 723.50 & 79.50 \\
\hline 11 & JLP - 4 & 750 & 696.50 & 105.80 \\
\hline 12 & ЛLР - 236 & 840 & 680.00 & 94.90 \\
\hline 13 & JLP - 235 & 856 & 644.10 & 81.40 \\
\hline 14 & JLP - 94 & 594 & 633.50 & 112.70 \\
\hline 15 & ЈLP - 83 & 824 & 614.00 & 91.50 \\
\hline 16 & JLP - 43 & 566 & 609.30 & 98.80 \\
\hline 17 & JLP - 64 & 864 & 597.60 & 78.60 \\
\hline 18 & JLP - 220 & 1395 & 582.70 & 57.90 \\
\hline 19 & JLP - 244 & 415 & 535.00 & 145.50 \\
\hline 20 & JLP - 201 & 575 & 516.20 & 90.40 \\
\hline PRO & MEDIO & 945 & 846.46 & 101.55 \\
\hline
\end{tabular}

En la Tabla $\mathrm{N}^{\circ} 2$, se reporta la producción de semillas de 20 genotipos correspondiente a la campaña 2008.

Tabla $\mathrm{N}^{\circ}$ 3: Producción de semillas y número de semillas por genotipo. Tacna 2009

\begin{tabular}{|c|c|c|c|c|}
\hline \multicolumn{2}{|c|}{ Orden Genotipo } & $\begin{array}{l}\mathbf{N}^{0} \text { de } \\
\text { sem. }\end{array}$ & Peso & $\begin{array}{c}\text { Peso } 100 \\
\text { Sem. }\end{array}$ \\
\hline 1 & JLP - 45 & & 1414.73 & 72.66 \\
\hline 2 & JLP - 57 & 1080 & 1242.02 & 114.98 \\
\hline 3 & JLP - 25 & 1282 & 1216.15 & 94.88 \\
\hline 4 & JLP - 1 & 1263 & 882.87 & 69.88 \\
\hline 5 & JLP - 202 & 822 & 789.26 & 96.07 \\
\hline 6 & JLP - 53 & 781 & 772.80 & 98.92 \\
\hline 7 & JLP -16 & 848 & 754.66 & 88.95 \\
\hline 8 & ЛLP -10 & 825 & 706.27 & 85.62 \\
\hline 9 & JLP - 64 & 583 & 696.61 & 119.49 \\
\hline 10 & ЛLP - 235 & 741 & 689.72 & 93.09 \\
\hline 11 & JLP - 24 & 680 & 682.70 & 100.34 \\
\hline 12 & JLP - 210 & 697 & 650.16 & 93.25 \\
\hline 13 & JLP -60 & 719 & 626.89 & 87.18 \\
\hline 14 & JLP - 4 & 672 & 614.21 & 91.40 \\
\hline 15 & JLP - 115 & 895 & 612.70 & 68.42 \\
\hline 16 & JLP - 2 & 558 & 603.70 & 108.24 \\
\hline 17 & JLP - 94 & 667 & 561.46 & 84.18 \\
\hline 18 & JLP - 236 & 803 & 554.40 & 69.04 \\
\hline 19 & JLP - 83 & 623 & 547.18 & 87.79 \\
\hline 20 & JLP - 43 & 669 & 544.82 & 81.48 \\
\hline PRO & MEDIO & 858 & 758.17 & 90.29 \\
\hline
\end{tabular}


Los rendimientos en promedio de los 20 genotipos fueron de $846.49 \mathrm{~g}$ y alcanzaron un máximo de 1737.50 $\mathrm{g}$, en el genotipo JLP-202, seguido por el genotipo JLP25 con 1720.40 g y el JLP-57 con 1295.40 .

En cuanto al peso de las semillas hay un promedio de $101.55 \mathrm{~g}$ con un rango que fluctúa entre 57.90 (JLP220) a 145.50 para JLP- 244

En la Tabla ํo 3, se reporta la producción de semillas de 20 genotipos correspondiente a la campaña 2009.

Los rendimientos en promedio de los 20 genotipos fueron de $758.17 \mathrm{~g}$ y alcanzaron un máximo de 1414.73 $\mathrm{g}$, en el genotipo JLP-45, seguido por el genotipo JLP-57 con $1242.02 \mathrm{~g}$ y en tercer lugar el JLP 25 con $1216.15 \mathrm{~g}$

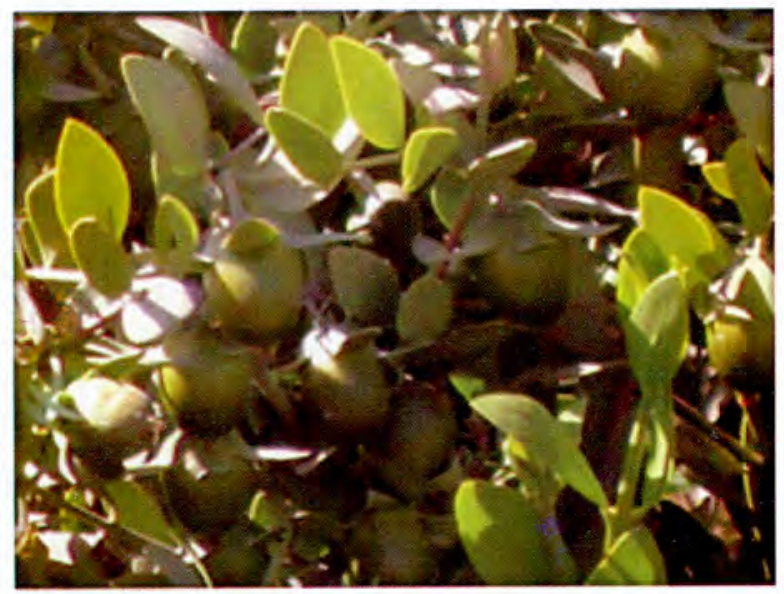

Figura $\mathrm{N}^{\circ}$ 4.Fructificación en brotes nuevos

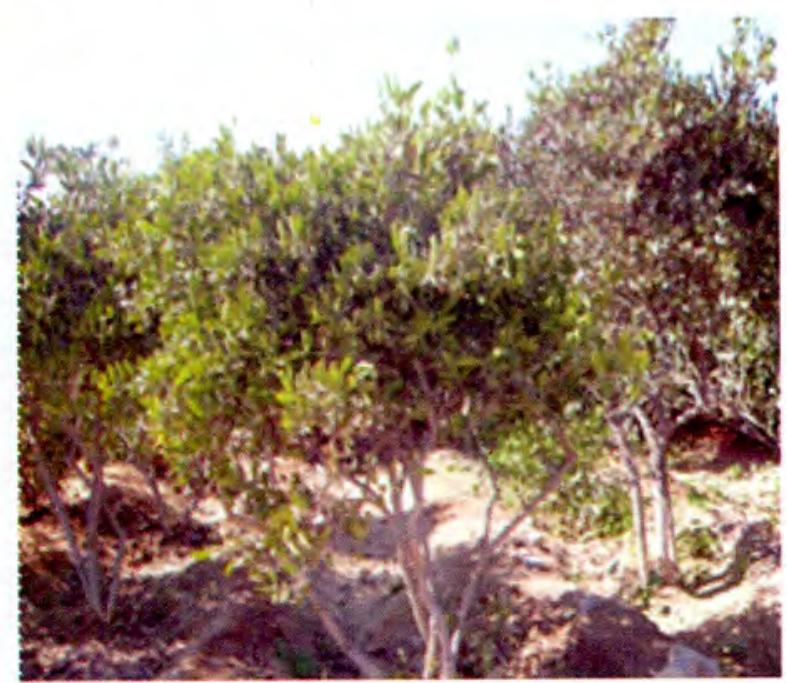

Figura $\mathrm{N}^{\circ}$ 5.Genotipo de jojoba buena arquitectura

\subsection{Evaluación de clones}

En la Tabla $\mathrm{N}^{\circ} 4$, se observa que el promedio en peso de semillas de los clones es de 208.40g. El clon C5-46 tiene el mayor peso con $545.60 \mathrm{~g}$, seguido del clon C2-21 con $447.20 \mathrm{~g}$.

Estos clones están iniciando su producción de semillas. Se espera que a partir del 6 año de producción los rendimientos sean superiores.

En la Tabla $\mathrm{N}^{\circ} 5$, se reportan los resultados de 20 clones de mayor producción de semillas durante el año 2009, siendo el promedio de los clones 287.04. g. El clon C111 obtuvo el mayor peso de semillas con $820.80 \mathrm{~g}$, seguido del clon C5-46 con $545.60 \mathrm{~g}$, y en tercer lugar el clon $\mathrm{C} 2-21$ con $411.75 \mathrm{~g}$.

Tabla $\mathbf{N}^{0}$ 4. Producción de semillas y número de semillas por clones. Tacna 2008

\begin{tabular}{cccc}
\hline Orden & Clon & $\begin{array}{c}\mathbf{N}^{\circ} \mathbf{D e} \\
\text { Sem. }\end{array}$ & $\begin{array}{c}\text { Peso } \\
\text { Sem. (g) }\end{array}$ \\
\hline 1 & C5 -46 & 769 & 545.6 \\
2 & C2 -21 & 442 & 447.2 \\
3 & C5 -47 & 626 & 300.7 \\
4 & C5 -52 & 421 & 298.6 \\
5 & C4 -43 & 357 & 288.2 \\
6 & C2 -12 & 365 & 234.5 \\
7 & C4 -45 & 252 & 229.6 \\
8 & C1 -3 & 229 & 182.1 \\
9 & C5 -50 & 210 & 167 \\
10 & C1 -1 & 211 & 158.3 \\
11 & C5 -54 & 169 & 158.1 \\
12 & C1 -9 & 185 & 145.3 \\
13 & C5 -49 & 173 & 142.4 \\
14 & C4 -40 & 153 & 139.8 \\
15 & C1 -10 & 49 & 136.6 \\
16 & C4 -37 & 153 & 134.4 \\
17 & C3 -26 & 136 & 119.4 \\
18 & C5 -53 & 130 & 115.8 \\
19 & C2 -51 & 138 & 114.7 \\
20 & C4 -44 & 427 & 109.7 \\
PROMEDIO & 279.75 & 208.4 \\
\hline
\end{tabular}

Tabla $\mathbf{N}^{\mathbf{0}}$ 5. Producción de semillas y número de semillas por clones. Tacna 2009

\begin{tabular}{cccc}
\hline Orden & Clon & $\begin{array}{c}\mathbf{N}^{\circ} \text { De } \\
\text { Sem. }\end{array}$ & $\begin{array}{c}\text { Peso } \\
\text { Sem. (g) }\end{array}$ \\
\hline 1 & C-1-11 & 1281 & 820.80 \\
2 & C- $5-46$ & 769 & 545.60 \\
3 & C-2-21 & 743 & 411.75 \\
4 & C- $4-44$ & 427 & 409.70 \\
5 & C- $1-1$ & 424 & 349.65 \\
6 & C- $5-47$ & 491 & 337.50 \\
7 & C- $5-53$ & 430 & 330.00 \\
8 & C- $2-18$ & 508 & 290.25 \\
9 & C- $4-43$ & 357 & 288.20 \\
10 & C- $4-34$ & 362 & 260.55 \\
11 & C- $4-35$ & 259 & 220.05 \\
12 & C- $2-17$ & 559 & 193.05 \\
13 & C- $1-3$ & 229 & 182.10 \\
14 & C- $1-2$ & 298 & 178.20 \\
15 & C- $2-12$ & 288 & 178.20 \\
16 & C- $4-45$ & 313 & 164.70 \\
17 & C- $5-49$ & 197 & 159.30 \\
18 & C- $5-55$ & 294 & 145.80 \\
19 & C- $1-7$ & 305 & 137.70 \\
20 & C- $2-20$ & 263 & 137.70 \\
PROMEDIO & 439.85 & 287.04 \\
\hline
\end{tabular}




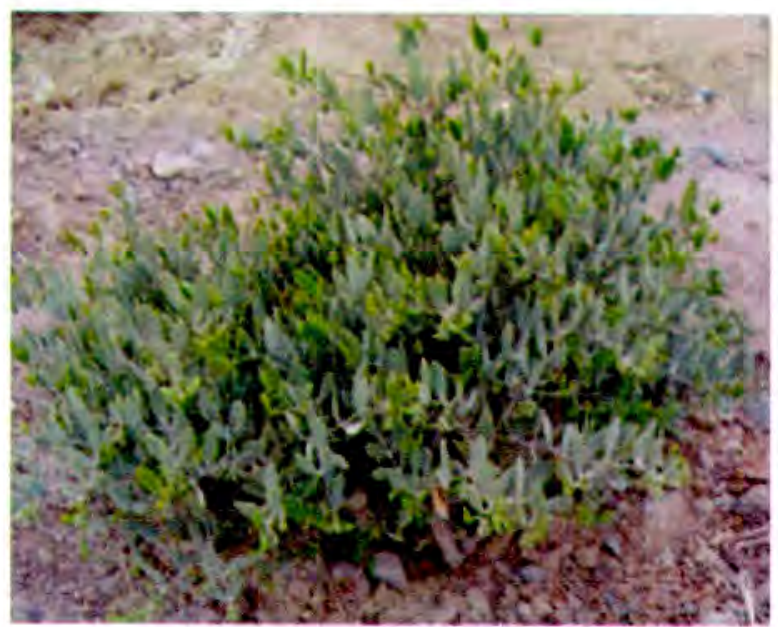

Figura ${ }^{\circ}{ }^{6}$. Clon C-1-11

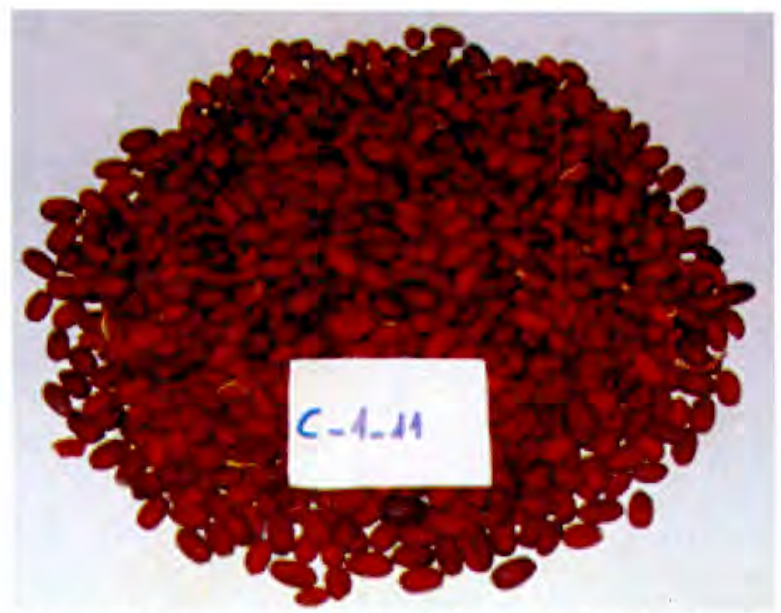

Figura $\mathrm{N}^{\circ}$ 7.Semillas del clon seleccionado C-1-11

\section{DISCUSIÓN}

\section{Evaluación Morfológica}

Al efectuar la evaluación morfológica en los genotipos de jojoba se observa que hay una gran variabilidad en altura de planta, posición de flores y frutos, forma y tamaño de frutos, forma de hojas, área foliar, vigor y arquitectura de planta esto debido a su tipo de propagación sexual y ser una planta dioica, tal como se observa en el Tabla $\mathrm{N}^{\circ} 1$ y Figuras 1, 2 y 3.

\section{Producción de Semillas en Genotipos de Jojoba}

La producción de semillas de los 20 genotipos fueron en promedio en el año 2009 de $758.17 \mathrm{~g}$ y alcanzaron un máximo de $1414.73 \mathrm{~g}$, en el genotipo JLP-45, seguido por el genotipo JLP-57 con $1242.02 \mathrm{~g}$ y en tercer lugar el JLP 25 con $1216.15 \mathrm{~g}$.

Los rendimientos en plantas de 8 años de edad fueron superiores a los reportados por la literatura para Israel y México. Así en Israel las plantas de 8 años de edad y con precipitación promedio de $204 \mathrm{~mm} / \mathrm{año}$; los rendimientos / plantas fueron $130 \mathrm{~g}$ con un máximo de $225 \mathrm{~g}$. En México en plantas de 6 y 7 años de edad, con precipitación de $190 \mathrm{~mm}$ y riego hasta alcanzar $500 \mathrm{~mm}$, la producción por planta fue de $200 \mathrm{~g}$ y $346 \mathrm{~g}$, respectivamente, en Estados Unidos de Norteamérica, Universidad de California, bajo condiciones de riego y con plantas de 6 y 7 años de edad, los rendimientos obtenidos fueron de $316 \mathrm{~g}$, con máximo de $1864 \mathrm{~g}$, en plantas de 6 años y de $691 \mathrm{~g}$, y con un máximo de 2073 $\mathrm{g}$, en plantas de 7 años.

Al comparar los resultados de este ensayo, con la literatura, los rendimientos obtenidos son superiores a los obtenidos en Israel y México, pero ligeramente inferiores a los de Estados Unidos, estos resultados obtenidos en este experimento, nos estaría indicando la posibilidad de adaptación del cultivo a las condiciones árido salinas de Tacna y la existencia de un potencial productivo de la especie si se realiza un programa de Mejoramiento además los resultados obtenidos se atribuyen a que los genotipos provienen de plantas madres previamente seleccionadas.

Los rendimientos obtenidos en el año 2009 se debería al efecto de la escases del agua lo que indujo a una ligera disminución del rendimiento con respecto al año 2008. Cabe indicar que se ha observado alternancia, en la producción de semillas de los genotipos es decir que hay años con buena producción y años con baja producción.

\section{Producción de Semillas en Clones de Jojoba}

En el Tabla $\mathrm{N}^{\circ} 7$, se reportan los resultados de 20 clones de mayor producción de semillas durante el año 2009 , siendo el promedio de los clones 287.04. g. El clon C111 obtuvo el mayor peso de semillas con $820.80 \mathrm{~g}$, seguido del clon C5-46 con $545.60 \mathrm{~g}$, y en tercer lugar el clon $\mathrm{C} 2-21$ con $411.75 \mathrm{~g}$.

La densidad en plantas propagadas vegetativamente son mayores que las propagadas sexualmente, razón por la cual la producción por semillas es mayor y la cosecha que estamos reportando es de plantas que están iniciando su producción.

También observamos mayor población de plantas productoras en las plantaciones propagadas vegetativamente porque se puede manejar la relación macho (polinizante): hembra. Es necesario resaltar que los clones al igual que los genotipos presentan la alternancia en la producción de semillas (vecería).

\section{V.CONCLUSIONES}

1. Existe variabilidad en la producción de semillas de los genotipos seleccionados, los cuales muestran atributos agronómicos deseables, tales como resistencia a plagas y enfermedades, buena arquitectura y tolerantes a suelos árido salinos.

2. Los genotipos de mayor producción de semillas bajo las condiciones árido salinas de Tacna en el 2009 alcanzaron un máximo de $1414.73 \mathrm{~g}$, en el genotipo JLP45 , seguido por el genotipo JLP-57 con $1242.02 \mathrm{~g}$ y en tercer lugar el JLP 25 con $1216.15 \mathrm{~g}$, los cuales fueron seleccionados para ser clonados.

3. El clon C1-11 obtuvo el mayor peso de semillas con 
820.80 g, seguido del clon C5-46 con $545.60 \mathrm{~g}$, y en tercer lugar el clon $\mathrm{C} 2-21$ con $411.75 \mathrm{~g}$.

\section{REFERENCIAS BIBLIOGRÁFICAS}

Allard, R.W. 1967. Principios de la Mejora Genética de las Plantas. Iera Edición Barcelona, Omega S.A 500 pp.

Arévalo S-N-1999. Evaluación de poblaciones y material segregante de Jojoba Sinmondsia chinensis Link Scheneider. En condiciones árido salinas de Tacna. Revista Agricultura Facultad de Ciencias Agrícolas de la Universidad Nacional Jorge Basadre Grohmann de Tacna.

Azocar, p, y Covarrubias, G. 1990: Adaptación de la Jojoba Sinmondsia chinensis Link (Scheneider), en condiciones de Azocar secado de la IV Región de Coquimbo Simiente- Vol $60-\mathrm{N}^{\circ} 2$

Castellanos, J. 2007. La jojoba un cultivo para zonas árido o desérticas. La Educación Agrícola. La agronomia alimenta al mundo.

Figuerola, P. 2006. Evapotranspiración del cultivo de la jojoba. INTA. Argentina.

FONDEF, 2008. La jojoba mucho más que un cultivo rentable. Santiago, Chile.
Hogan, L y W.P. Bemis. 1983: Búfalo gourd and jojoba. Potencial new crops for arid lanas. Advances in Agronomics 36:317-349.

Ortiz, J.M. y Ladux, J.L. 2006. Cultivo de la jojoba. Agencia de Extensión Rural INTA La Rioja, Argentina.

Palzkill, D.A y Hogan, L. 1983: Studies indicate early material selecction may increase production Jojoba Happenings. Phoenix, Arizona. USA II(2):1-1-6.

Ricks, D.J. 2004. El aceite de jojoba en cosméticos. Guia del Emprendedor.com.ar:

Rudolph, N.G. 1983: Ensayos de Técnicas en vivero para la producción de plantas de Jojoba Sinmondsia chinensis (Link) Schneider. IDESIA (CHILE) Vol.7.

Yermanos, D:M.1983: Perfomance of Jojoba Ander cultivation between 1973-1982: Information developed at et University of California, Riverside. Report, 13 pp.

Correspondencia:

Nelly Arevalo Solsol

Ciudad Universitaria "Los Granados"

Av. Miraflores s/n. Tacna. Perú

arevalonelly@hotmail.com 